\title{
Comparison of the effects of 23-gauge and 25 -gauge microincision vitrectomy blade designs on incision architecture
}

This article was published in the following Dove Press journal:

Clinical Ophthalmology

19 November 2014

Number of times this article has been viewed

\author{
Makoto Inoue' \\ Dina Joy K Abulon² \\ Akito Hirakata' \\ 'Kyorin Eye Center, School \\ of Medicine, Kyorin University, \\ Tokyo, Japan; ${ }^{2}$ Alcon Research, Ltd., \\ Irvine, CA, USA
}

Correspondence: Makoto Inoue Kyorin Eye Center, School of Medicine, Kyorin University, 6-20-2 Shinkawa, Mitaka, Tokyo, I8I-86II, Japan Tel +8I 422475 II I ext 2606 Fax +8I 422469309

Email inoue@eye-center.org
Purpose: To compare the effects of different 23- and 25-gauge microincision vitrectomy trocar cannula entry systems on incision architecture.

Methods: We tested one ridged microvitreoretinal (MVR), one non-ridged MVR, one pointed beveled, and one round-tipped beveled blade ( $n=10$ per blade design per incision type). Each blade's straight and oblique incision architecture was assessed in a silicone disc simulating the sclera. Wound leakage under pressure and endoscopic observations were conducted on sclerotomy sites of isolated porcine eyes ( $\mathrm{n}=4$ per blade design) after simulated vitrectomy.

Results: Differences in blade design created distinct incision architecture. Incisions were linear with the ridged MVR blade, flattened "M-shaped" with the non-ridged MVR blade, asymmetrical chevron-shaped with the pointed beveled blade, and curved with the round-tipped beveled blade. With the exception of oblique entry incision thickness, both MVR blade designs created thinner incisions than the beveled blades at entry and exit sites. Only the ridged MVR blade created incisions with no leakage. Vitreous incarceration was observed with all trocar cannula systems.

Conclusion: Wound closure in porcine eyes was similar with all blades despite differences in incision architecture. Wound leakage occurred at low to moderate infusion pressures with most blades; no wound leakage was observed with ridged MVR blades.

Keywords: entry system, incision closure, leakage, pars plana incision, sclerotomy, trocar blade

\section{Introduction}

Microincision vitrectomy surgery (MIVS), also referred to as transconjunctival sutureless vitrectomy, ${ }^{1}$ is widely used to treat vitreoretinal pathologies. ${ }^{2,3}$ The use of sutureless sclerotomies with conventional beveled entry systems decreases surgeryinduced astigmatism and reduces visual recovery time. ${ }^{4}$ The 23 -gauge sutureless vitrectomy, first described by Eckardt in $2005,{ }^{5}$ consists of creating a slit-shaped sclerotomy by inserting a microvitreoretinal (MVR) trocar or blade at an angle of approximately $30^{\circ}$, followed by placement of a cannula in the sclerotomy incision using a blunt-tipped inserter. The 23-gauge instruments are then passed into the vitreous through the cannula.

Microincision vitrectomy surgical techniques have evolved from a two-step procedure to a one-step procedure with 23- or 25-gauge microcannula and beveled-blade incisions. ${ }^{1,5}$ Good results have been observed with small-gauge entry systems, ${ }^{6,7}$ although hypotony due to leakage, choroidal detachments, and endophthalmitis have been reported as postoperative complications. ${ }^{2}$ An oblique sclerotomy (ie, an angled or oblique incision) made with a 23 - or 25 -gauge system produces minimal wound leakage. ${ }^{8-10}$ Histologic 
and optical coherence tomography (OCT) analysis of oblique incision wounds from 23- and 25-gauge systems has shown better wound closure, reduced leakage of intraocular fluid, and blocked entry of India ink from the surface of the eye into the incision compared with vertical (ie, straight) incisions. ${ }^{11}$

A higher incidence of postoperative endophthalmitis was reported after 25-gauge vitrectomy compared with 20-gauge vitrectomy. ${ }^{12,13}$ In studies using oblique incisions for MIVS, there were no significant differences in the incidence of postoperative endophthalmitis among the 25-gauge MIVS, 23-gauge MIVS, and conventional 20-gauge vitrectomy cases. ${ }^{14-16}$ Histologic analysis of human cadaver and animal eyes showed that oblique incisions led to better wound closure than straight incisions. ${ }^{17,18}$ However, transient postoperative hypotony after MIVS may allow fluid on the ocular surface to enter the eye and introduce bacterial contamination, resulting in endophthalmitis. ${ }^{17,19-21}$ Thus, guidelines have been presented on how to reduce the risk of hypotony or endophthalmitis associated with MIVS; these guidelines include use of oblique incisions. ${ }^{3}$

Incision architecture also plays an important role in MIVS outcomes. As discussed by Arevalo et al suboptimal wound shape can contribute to endophthalmitis and retinal breaks. ${ }^{22}$ It has also been suggested that slit-shaped wounds exhibit better closure, and therefore less risk of leakage, compared with chevron-shaped wounds created using traditional round trocar blades. ${ }^{22}$

The aim of this study was to evaluate how the design of currently available MIVS entry systems affects incision architecture and wound closure in simulated sclerotomies.

\section{Materials and methods}

Seven trocar cannula systems of different gauges and designs were studied (Table 1); tested blades included one MVR blade with a tapered blade and cylinder ridge (ie, ridged MVR; EDGEPLUS ${ }^{\circledR}$; Alcon Laboratories, Inc., Fort Worth, TX, USA), one non-ridged MVR blade (MANI, Inc., Utsunomiya, Japan), a pointed beveled blade (Alcon Laboratories, Inc.), and a round-tipped beveled blade (Dutch Ophthalmic Research Center International, Inc., BV, VN Zuidland, Netherlands). A scanning electron microscope
(SEM) was used to examine the cutting surfaces of each blade. High-magnification imaging was used to measure the geometry of straight and oblique incisions made through circular silicone discs. Leakage testing was conducted on the sclerotomy sites after simulated vitrectomy in enucleated porcine eyes. Video recordings captured the creation of the sclerotomy incisions from inside the eye.

\section{SEM analyses of blade cutting surface}

After removal of the plastic handle, each trocar blade $(n=1$ per design) was mounted on conductive carbon tape. The cutting edge was examined using an AMRAY 1610 SEM (AMRAY, Inc., Bedford, MA, USA).

\section{Measurements of incision shapes}

For each blade type, ten straight and ten oblique incisions were assessed, with a new blade used for each incision to avoid confounding effects of blade dulling. An incision was created with each type of blade through a silicone disc that was secured to a metal plate below a mount holding each type of blade (Figure 1A and B). The Instron Single Test Column System (Model 5542; Instron, Norwood, MA, USA) was lowered until the blades completely penetrated the silicone disc. Using a novel method of assessing simulated incision geometry, straight $\left(90^{\circ}\right.$ angle) and oblique ( $30^{\circ}$ angle) incisions were created by rotating the mounted silicone disc $90^{\circ}$ and $30^{\circ}$ relative to the blade, respectively. A bevel-down direction was used for the pointed beveled and round-tipped beveled blades. Two illuminated optic fibers were placed on opposite sides of the silicone disc to transilluminate the incision site during blade penetration.

The width and thickness of incisions at the entry and exit sites were assessed using a digital measuring instrument and associated software (Optical Gauging Products, Rochester, NY, USA). Incision endpoints were marked with crosshairs using the Optical Gauging Products software, which then calculated the distance between endpoints (ie, incision width) and the length of incision deviation from a virtual straight line connecting the endpoints (ie, incision thickness; Figure 2A). Incision width and thickness were related to the

Table I Microincision vitrectomy blades evaluated

\begin{tabular}{|c|c|c|}
\hline Trocar cannula system & Gauges tested & Manufacturer \\
\hline Ridged MVR blade (EDGEPLUS ${ }^{\circledR}$ ) & 23,25 & Alcon Laboratories, Inc., Fort Worth, TX, USA \\
\hline Non-ridged MVR blade & 23,25 & MANI, Inc., Utsunomiya, Japan \\
\hline Pointed beveled blade & 23,25 & Alcon Laboratories, Inc., Fort Worth, TX, USA \\
\hline Round-tipped beveled blade & 23 & Dutch Ophthalmic Research Center International, Inc., BV, VN Zuidland, the Netherlands \\
\hline
\end{tabular}

Abbreviation: MVR, microvitreoretinal. 

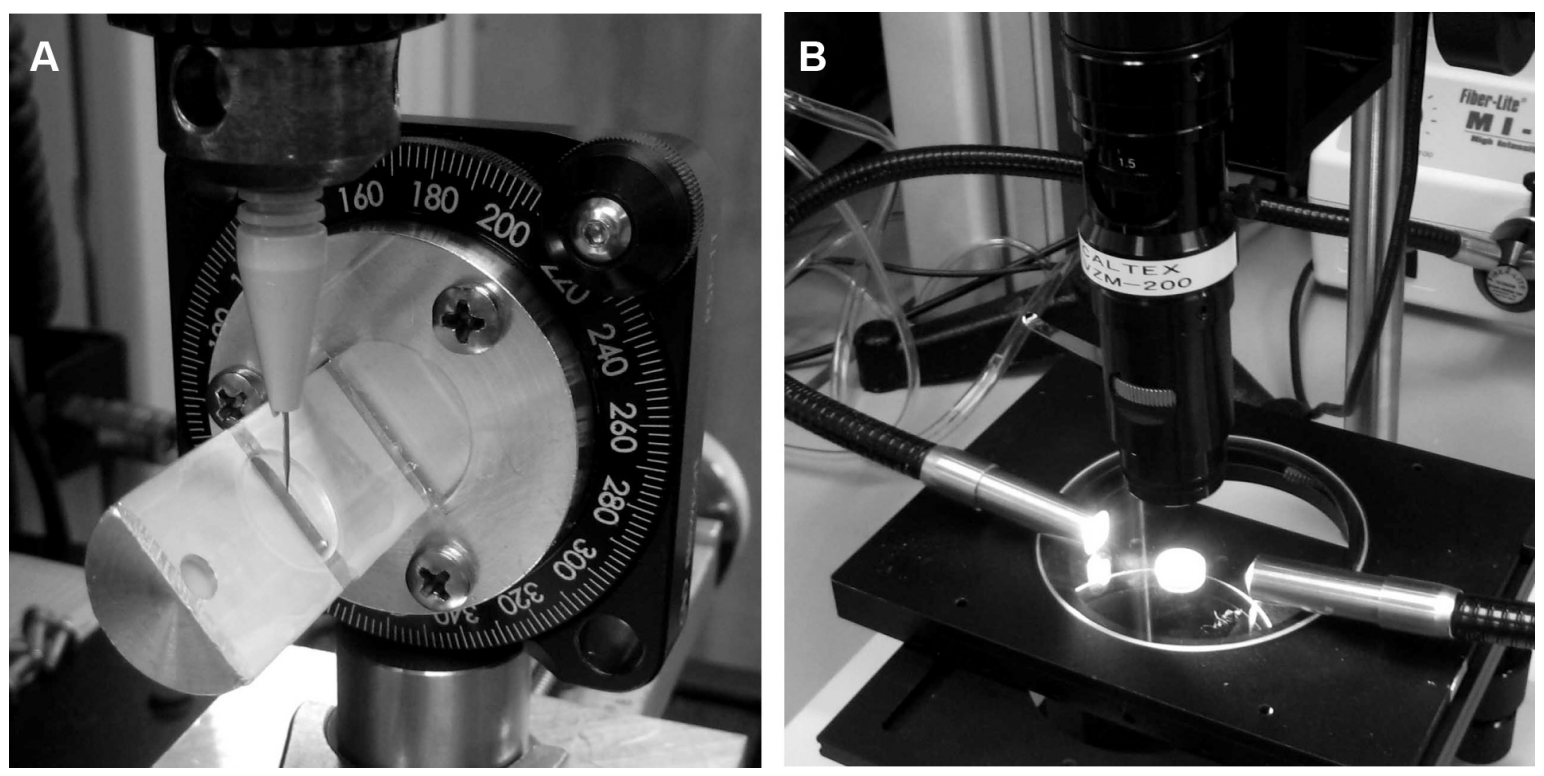

Figure I Photographs of blade penetration and observation of wound structure.

Notes: (A) A I0-mm silicone disc was mounted at $30^{\circ}$ relative to the blade. (B) Transillumination made the passage of the blade penetrating the plate more visible.
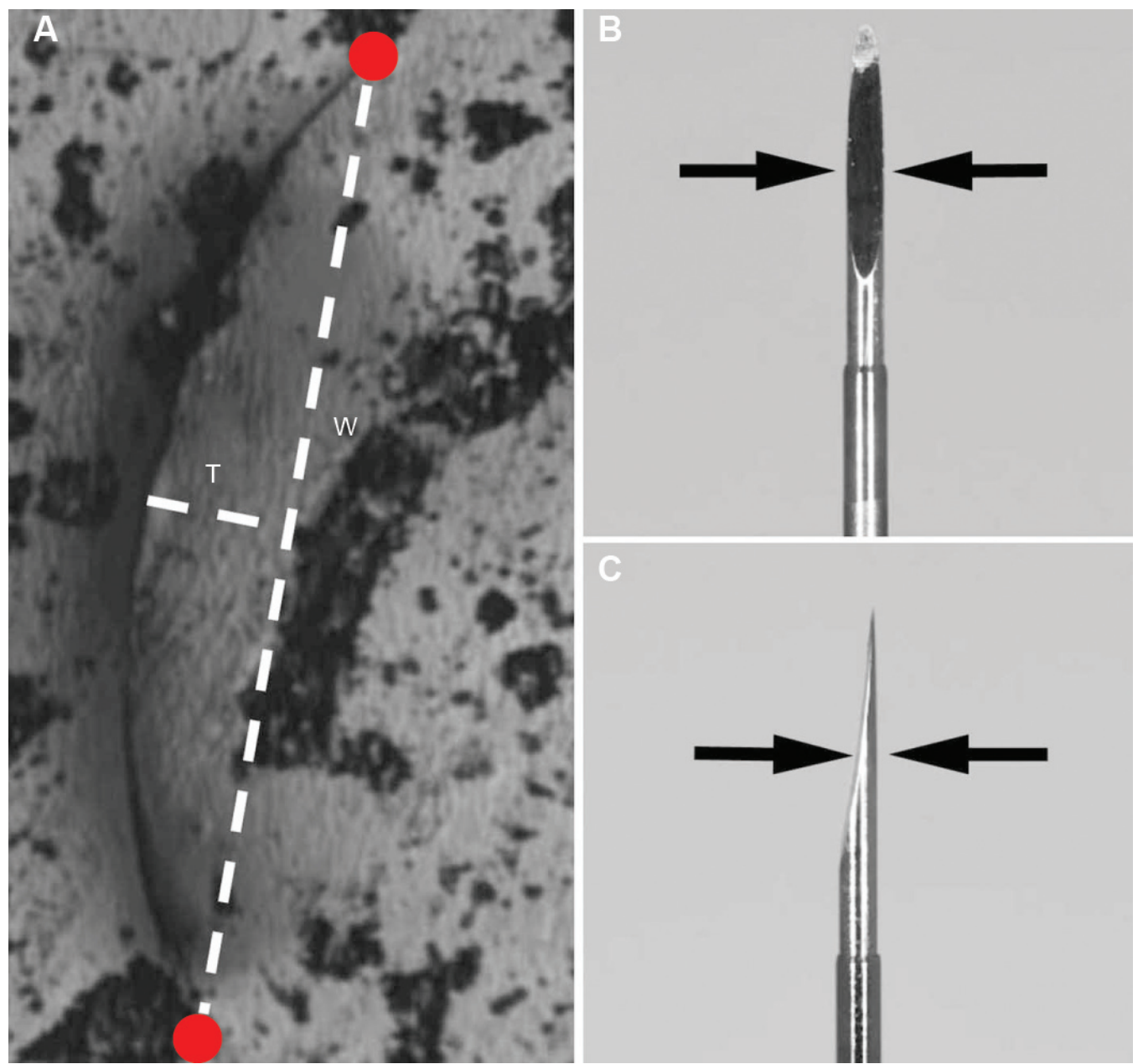

Figure 2 Definition of the incision thickness and width.

Notes: (A) The incision thickness (T) and width (W) at the entry and exit sites as calculated by digital measuring software. Red dots indicate incision endpoints. (B) The incision width (indicated by arrows) was related to the width of the blade. (C) The incision thickness (indicated by arrows) was related to the thickness and design of the blade. 
width (Figure 2B) and the thickness of the blade (Figure 2C), respectively. The incision entry site corresponded to the conjunctival side of the eye, and the exit site corresponded to the side of the vitreous. Incision thickness (ie, deviation from an ideal straight line between endpoints of the incision width) was calculated using the maximum deviation distance from ideal straightness.

\section{Wound leakage}

Twenty-eight fresh porcine eyes obtained from a local abattoir were used for the wound leakage experiments $(n=4$ per blade design and gauge). For each test, eyes were mounted in the same orientation and, to realistically model surgical technique, incisions were created manually by a single investigator (MI). The conjunctiva was displaced during incision creation. Each trocar cannula system was inserted obliquely through the pars plana in the same location for each eye; a bevel-down direction was used for the pointed beveled and round-tipped beveled blades. A protractor was used to ensure that incision angles were similar among eyes. Three incisions were created $3.0 \mathrm{~mm}$ posterior to the limbus for the infusion cannula, endoilluminator, and vitrectomy probe. The incision that was used for the vitrectomy probe was evaluated for leakage in each eye after plugs were applied to cannulas in the other incisions. Wound leakage was defined as a continuous leakage of fluid from the incision site. A complete core vitrectomy was performed with the core duty-cycle mode using the CONSTELLATION ${ }^{\circledR}$ Vision System (Alcon Laboratories, Inc.) at 5,000 cuts/ minute and $650-\mathrm{mmHg}$ vacuum pressure. Every effort was made to remove the vitreous in the periphery and around the cannula until free flow of irrigation solution out of the cannula was observed. To make potential leakage more visible, approximately $0.2 \mathrm{~mL}$ of $2.5-\mathrm{mg} / \mathrm{mL}$ indocyanine green solution was injected into the infusion line.

The nonvalved cannula used with the vitrectomy probe was removed from the sclera using the guidance of the vitrectomy probe shaft. After the cannulas were removed, the incision was massaged with a cotton swab for at least 10 seconds at $30-\mathrm{mmHg}$ infusion to seal the incision. After the incision was sealed, the conjunctiva was trimmed to enable visualization of any fluid leakage from the incision site. Using Castroviejo forceps, the conjunctiva near the targeted incision site was trimmed to create a 5- to 7-mm diameter hole. Assessment of wound closure by measuring leakage under increasing infusion pressure has been described previously. ${ }^{23,24}$ Using a novel approach in which infusion pressure was controlled by the vitrectomy machine, the infusion pressure was lowered to $0 \mathrm{mmHg}$ and increased in $5-\mathrm{mmHg}$ increments until leakage was observed. The infusion pressure at which wound leakage occurred was documented, and wound leakage was scored on a scale from one to six as follows: low infusion pressure (1: 0-23 $\mathrm{mmHg}$ ), moderate infusion pressure (2: 24-47 mmHg or 3: 48-71 $\mathrm{mmHg}$ ), moderate-high infusion pressure (4: 72-95 $\mathrm{mmHg}$ or $5: 96-119 \mathrm{mmHg}$ ), and high infusion pressure (6: $\geq 120 \mathrm{mmHg}$ ). A higher leakage score indicated less leakage (ie, better incision sealing).

\section{Endoscopic evaluation of inner sclerotomy sites}

Fourteen fresh porcine eyes obtained from a local abattoir were used for the inner sclerotomy-site experiments. An endoscopic attachment (VH-B18; KEYENCE Corporation, Osaka, Japan) was mounted to a digital microscope (VHX-1000; KEYENCE Corporation). The endoscope, with a rod lens $1.9 \mathrm{~mm}$ in diameter, was inserted through a posterior sclerotomy site and positioned such that the pars plana was visible. Three oblique incisions were created $3.0 \mathrm{~mm}$ posterior to the limbus, and the penetration of each blade was observed from inside the eye with the endoscope. As described above, porcine eyes were mounted in the same orientation, and incisions were manually created by a single investigator; incision angles were standardized using a protractor. The insertion and removal of the trocar cannula system were photographed through the endoscope.

\section{Statistical analysis}

Statistical analyses were performed using Microsoft Office Excel 2010 (Microsoft Corporation, Redmond, WA, USA) with Excel Statistics 2010 Add-On (Social Survey Research Information, Tokyo, Japan). The nonparametric KruskalWallis one-way analysis of variance was used to compare differences between data sets for incision thickness and width. If a statistical difference existed between at least two of the samples, the Scheffé test was applied to identify where the differences were for multiple comparisons. Wound leakage scores were compared between blade types using a Mann-Whitney $U$-test. $P<0.05$ was considered statistically significant. Values are expressed as mean \pm standard deviation.

\section{Results \\ SEM examinations of blades}

All blades had right-to-left symmetry; the ridged MVR blades also had unilateral front-to-back symmetry (Figure 3A-D). 

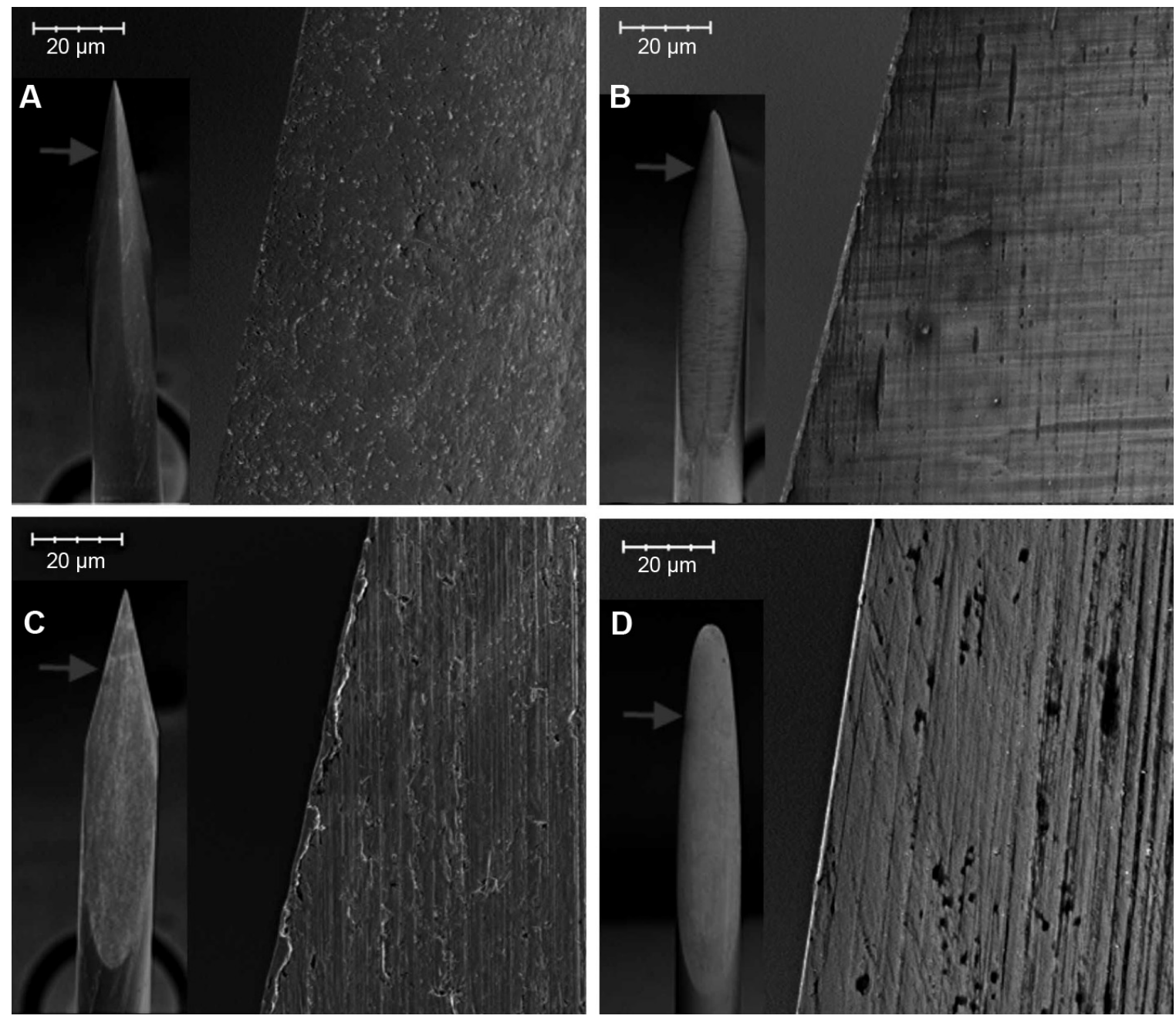

Figure 3 High-magnification images of 23-gauge blades near the tip by scanning electron microscopy.

Notes: (A) No striations were seen on the ridged MVR blade. (B) Note the burrs along the cutting edge of the pointed beveled blade and the surface striations. (C) Surface striations were observed on the cutting edge of the non-ridged MVR blade. (D) Surface striations were seen on the round-tipped beveled blade. The arrow shows the cutting edge (A-D).

Abbreviation: MVR, microvitreoretinal.

The pointed beveled blades and the round-tipped beveled blades had no unilateral (ie, front-to-back) symmetry. SEM images of the 23- and 25-gauge non-ridged MVR blades showed a quadrilateral facet near the tip (Figure 3C). With the exception of the ridged MVR blades, all blades had striations along the blade edge (Figure 3A-D). The beveled side of the pointed beveled blades had many burrs along the edge.

\section{Incision shape at entry and exit sites of the silicone disc}

Straight incision shapes with 23- and 25-gauge blades at the entry and exit sites were linear and slit-like with the ridged MVR blades; a flattened "M" shape with the non-ridged MVR blades; a chevron shape with the pointed beveled blades; and a curved shape with the round-tipped beveled blades (23-gauge only; Figure 4).
For oblique incisions with 23- and 25-gauge blades, both the ridged and non-ridged MVR blades created shallowangle, "V"-shaped incisions at the entry and exit sites; at the exit sites, the "V"-shape was inverted and the vertex was opposite the original direction of the entry sites (Figure 5). Both MVR blades generated a groove at the incision vertex. The shape of the incisions created by 23- and 25-gauge pointed and round-tipped beveled blades was round at the entry site and an acute-angle "V" shape at the exit site. The path of the round-tipped beveled incisions from the entry site to the exit site appeared longer than the incisions created by the other blades.

For both straight and oblique incisions, incision thickness was related to the straightness (ie, linearity of entry and exit sites) of the incision, with thinner incisions corresponding to smaller deviation from straightness. In general, there 


\section{3-gauge incision shapes}

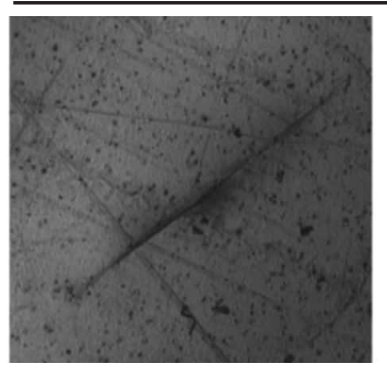

Ridged MVR

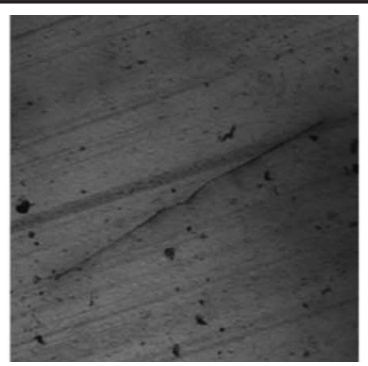

Non-ridged MVR

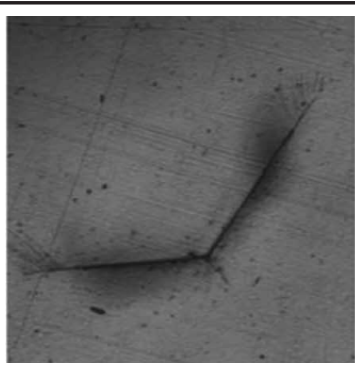

Pointed beveled

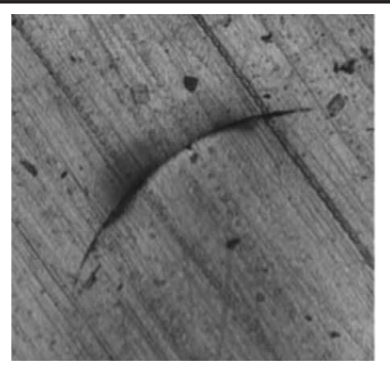

Round-tipped beveled

\section{5-gauge incision shapes}

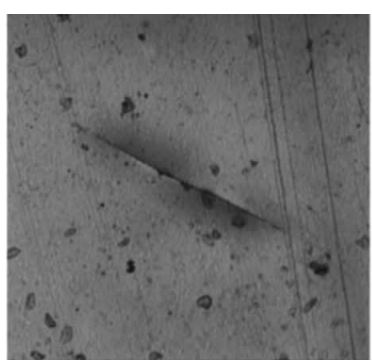

Ridged MVR

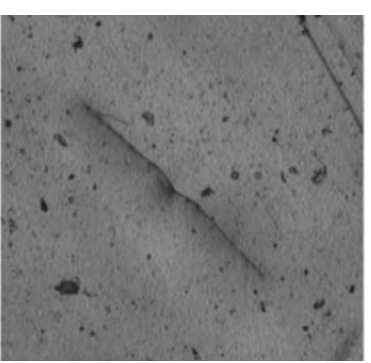

Non-ridged MVR

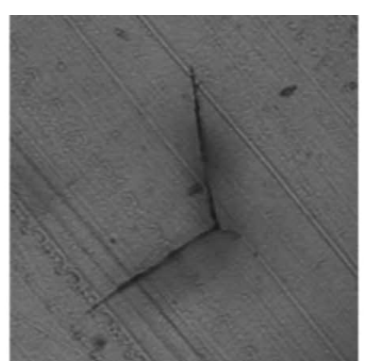

Pointed beveled

Figure 4 Incision shape of 23- and 25-gauge blades with straight incision at the entry site.

Notes: The incision shapes of 23- and 25-gauge blades were linear and slit-like with the ridged MVR blades, flattened "M"-shaped with the non-ridged MVR blades, chevronshaped with the pointed beveled blades, and curved with the round-tipped beveled blade.

Abbreviation: MVR, microvitreoretinal.

\section{3-gauge incision shapes}

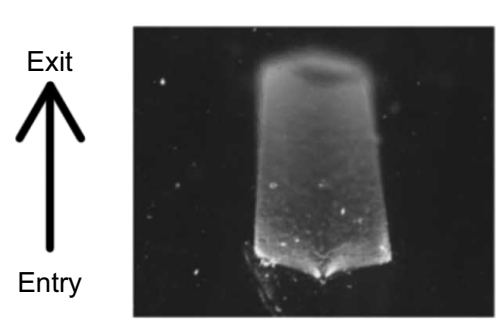

Ridged MVR

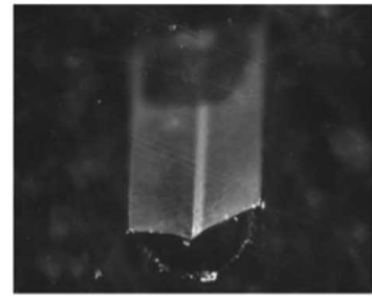

Non-ridged MVR

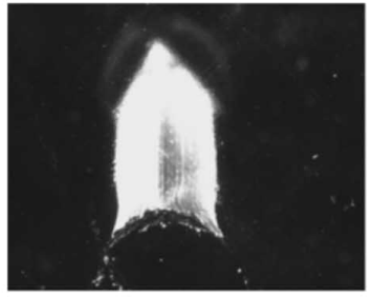

Pointed beveled

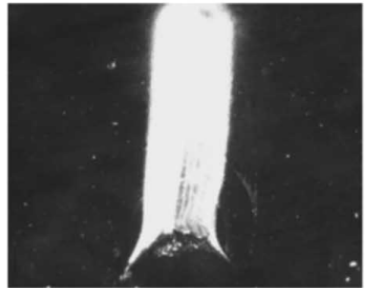

Round-tipped beveled

\section{5-gauge incision shapes}

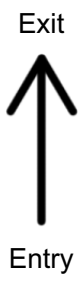

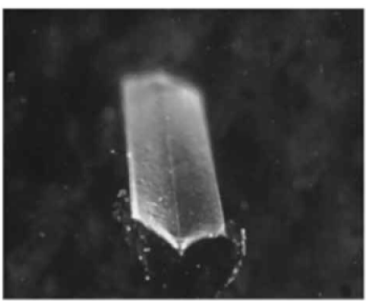

Ridged MVR

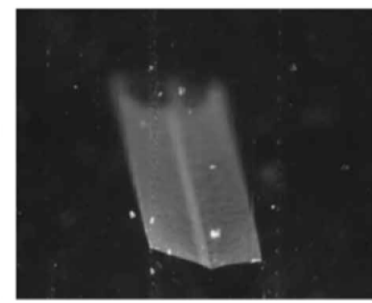

Non-ridged MVR

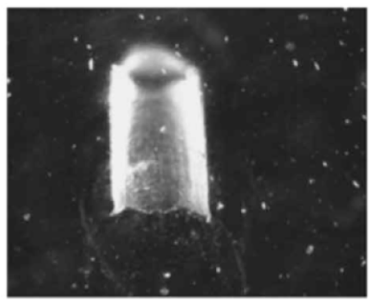

Pointed beveled

Figure 5 Incision shape of 23- and 25-gauge blades with oblique incision.

Notes: Both MVR blades (ie, ridged and non-ridged) created a shallow angled " $V$ "-shaped incision at entry and exit sites. Incisions with the pointed beveled and round-tipped beveled blades were round at the entry sites, with an acute angle " $\mathrm{V}$ "-shape at the exit sites.

Abbreviation: MVR, microvitreoretinal. 
were more significant differences among blades for oblique incisions versus straight incisions.

\section{Straight incision architecture}

With the exception of incision entry site width for 23-gauge blades, straight incision architecture at entry and exit sites was significantly different among gauge-matched blades $(P=0.038$ to $P<0.0001$; Kruskal-Wallis test; Table 2). Entry and exit site thickness was smallest with ridged MVR blades, and was greatest with pointed beveled blades. Incision thickness was generally similar with ridged versus non-ridged MVR blades. Incision width at entry and exit sites was less predictable.

For the 23-gauge blades, the thickness of entry and exit sites was greatest with pointed beveled blades, followed by round-tipped beveled, non-ridged MVR, and ridged MVR blades. Entry and exit thickness was significantly greater with pointed beveled blades compared with ridged or non-ridged MVR blades ( $P<0.001$ for both); entry site thickness was also significantly greater with round-tipped beveled blades compared with ridged MVR blades $(P<0.05)$. Entry site width was similar among all 23-gauge blades tested. Exit site width was smallest with pointed beveled and round-tipped beveled blades, followed by ridged MVR and non-ridged MVR blades. Exit site width was significantly greater with non-ridged MVR blades compared with pointed beveled blades $(P<0.05)$.

For 25-gauge blades, incision thickness at entry and exit sites was smallest with ridged MVR blades and greatest with pointed beveled blades. Compared with pointed beveled blades, entry site thickness was significantly smaller with ridged MVR blades $(P<0.001)$ and non-ridged MVR blades $(P<0.05)$. Similarly, exit site thickness was significantly smaller with ridged and non-ridged MVR blades compared with pointed beveled blades $(P<0.001$ and $P<0.05$, respectively); the thickness of exit sites was also significantly smaller with ridged MVR blades compared with non-ridged MVR blades $(P<0.05)$. Entry site width was similar between ridged and non-ridged MVR blades, and was significantly smaller with ridged MVR blades compared with pointed beveled blades $(P<0.05)$. Exit site width was greatest with non-ridged MVR blades, followed by ridged MVR blades and pointed beveled blades. The width of exit sites was significantly smaller with pointed beveled blades compared with non-ridged MVR blades $(P<0.001)$.

\section{Oblique incision architecture}

Oblique incision architecture was significantly different among gauge-matched blades $(P=0.0007$ to $P<0.0001$; Kruskal-Wallis test; Table 2). In general, ridged MVR blades created the thinnest incisions (ie, smallest thickness) at both the entry and the exit sites, followed by non-ridged MVR blades, round-tipped beveled blades, and pointed beveled blades. Incision width at entry and exit sites was more variable, as described below.

For the 23-gauge blades, entry site thickness was significantly greater with pointed beveled blades compared with ridged MVR blades or round-tipped beveled blades $(P<0.001$ for both), and with non-ridged MVR blades compared with round-tipped beveled blades $(P<0.05)$. Exit site thickness was markedly greater with pointed beveled blades compared with other blades tested and was significantly thicker compared with ridged MVR or non-ridged MVR blades $(P<0.001$ for both). Exit site thickness with ridged MVR blades was significantly smaller compared with round-tipped beveled blades $(P<0.05)$. Incision width at entry and exit sites was smallest with round-tipped beveled blades. Entry site width with round-tipped beveled blades was significantly smaller compared with non-ridged MVR blades $(P<0.001)$ or pointed beveled blades $(P<0.01)$. Exit site width with round-tipped beveled blades was significantly smaller compared with ridged MVR and non-ridged MVR blades ( $P<0.05$ for both). Exit site width was significantly greater with non-ridged MVR blades compared with pointed beveled blades $(P<0.001)$. There were no statistically significant differences between 23-gauge ridged and non-ridged MVR blades for incision thickness or width at entry or exit sites.

For 25-gauge blades, incision thickness at entry and exit sites was smallest with ridged MVR blades followed by nonridged and pointed beveled blades. Entry site thickness was significantly smaller with ridged MVR blades compared with non-ridged MVR blades $(P<0.05)$ or pointed beveled blades $(P<0.001)$, and with non-ridged MVR blades compared with pointed beveled blades $(P<0.05)$. Exit site thickness was significantly smaller with ridged and non-ridged MVR blades compared with pointed beveled blades $(P<0.001$ for both). Incision width at entry sites was significantly smaller with ridged MVR blades compared with pointed beveled or non-ridged MVR blades ( $P<0.05$ for both). At exit sites, incision width was smallest with pointed beveled blades. Incision width at exit sites was significantly greater with non-ridged MVR blades compared with ridged MVR blades $(P<0.05)$ or pointed beveled blades $(P<0.001)$.

\section{Wound leakage}

No leakage was observed for incisions created by the 23- and 25-gauge ridged MVR blade trocar cannula systems (Table 3). With the 23- and 25-gauge pointed beveled trocar 


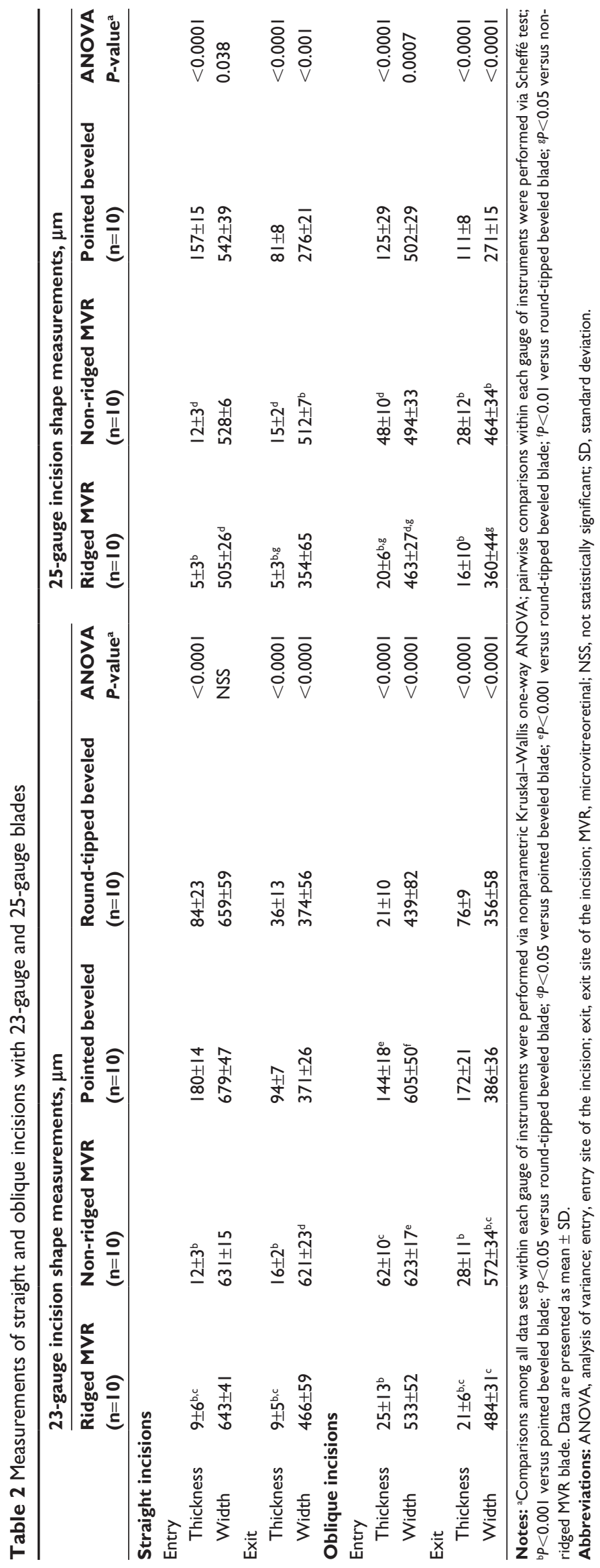




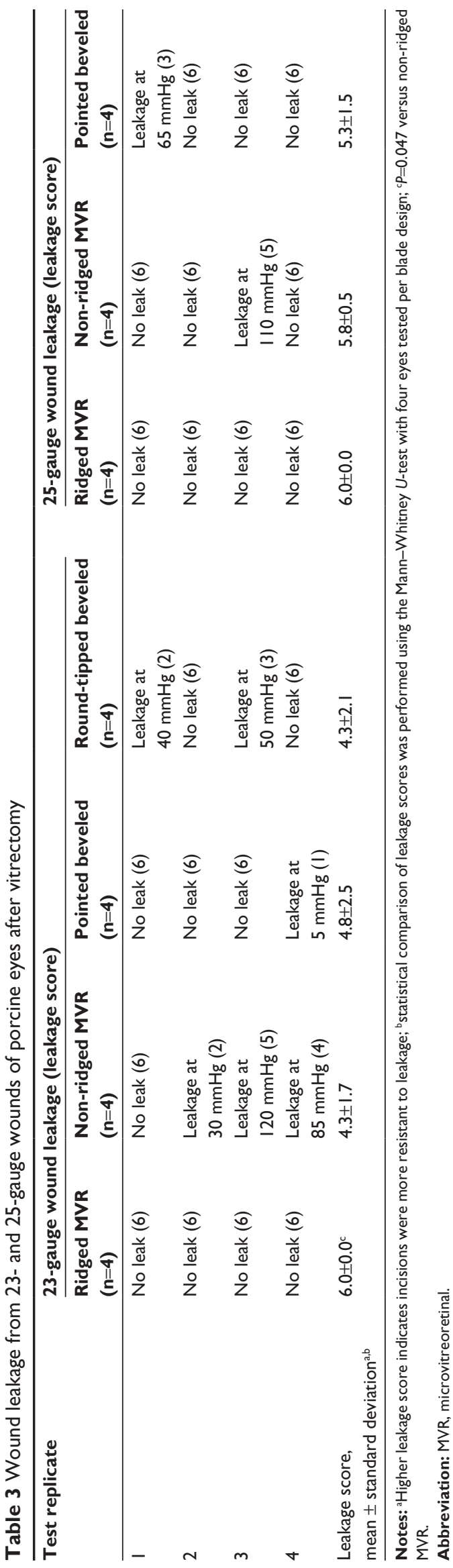

cannula systems, one out of four incisions leaked at low ( $5 \mathrm{mmHg}$ ) or moderate (30-65 $\mathrm{mmHg}$ ) observed infusion pressures (leakage scores of 1 or $2-3$, respectively). With the 23-gauge round-tipped beveled blades, two out of four incisions leaked at moderate infusion pressures. The stability of the non-ridged MVR blade incisions was highly variable, with leakage occurring in three out of four of the 23-gauge incisions at moderate to high infusion pressures (85-120 mmHg; leakage scores of 4-5) and in one out of four of the 25-gauge incisions at a high pressure.

The leakage score of the 23-gauge ridged MVR trocar cannula system was significantly higher than that of the 23-gauge non-ridged MVR trocar cannula system $(P=0.047$; Mann-Whitney $U$-test) but not the 23-gauge pointed beveled $(P=0.317)$ or the round-tipped beveled $(P=0.131)$ trocar cannula systems. The leakage score of the 25 -gauge ridged MVR trocar cannula was numerically higher than that of the 23-gauge round-tipped beveled trocar cannula, and was similar to leakage scores of the 25-gauge non-ridged MVR ( $P=0.317)$ and the pointed beveled $(P=0.317)$ trocar cannula systems.

\section{Endoscopic evaluation of inner sclerotomy sites}

Results of endoscopic evaluation of oblique incisions were similar for all trocar cannula entry systems; a representative example is shown in Figure 6A-C. When the blades were inserted, the endoscopic view showed a stretching of the ciliary epithelium. The elongated section of the ciliary epithelium was folded after the trocar blade was removed, and the cannula was inserted perpendicular to the sclera. When the cannula was removed, an incarceration of vitreous fibers was seen in the vitreous base. An incarceration of vitreous in the wound was observed with all entry systems; no specific blade had a higher degree of incarceration. After removal of the cannula, a slit-shaped defect of the ciliary epithelium was observed with all of the different entry systems. All oblique incisions appeared to seal in the same manner.

\section{Discussion}

The findings from this study show that each blade type had a unique design and created a distinct incision shape. All of the blades tested had a right-to-left symmetry; only the ridged MVR blades had a unilateral front-to-back symmetry and created linear, slit-like incisions.

The ridged MVR blades created the thinnest incisions using straight entry. In the case of oblique incisions, the round-tipped beveled blades created the thinnest incisions at the entry sites but produced a thicker incision at the exit 

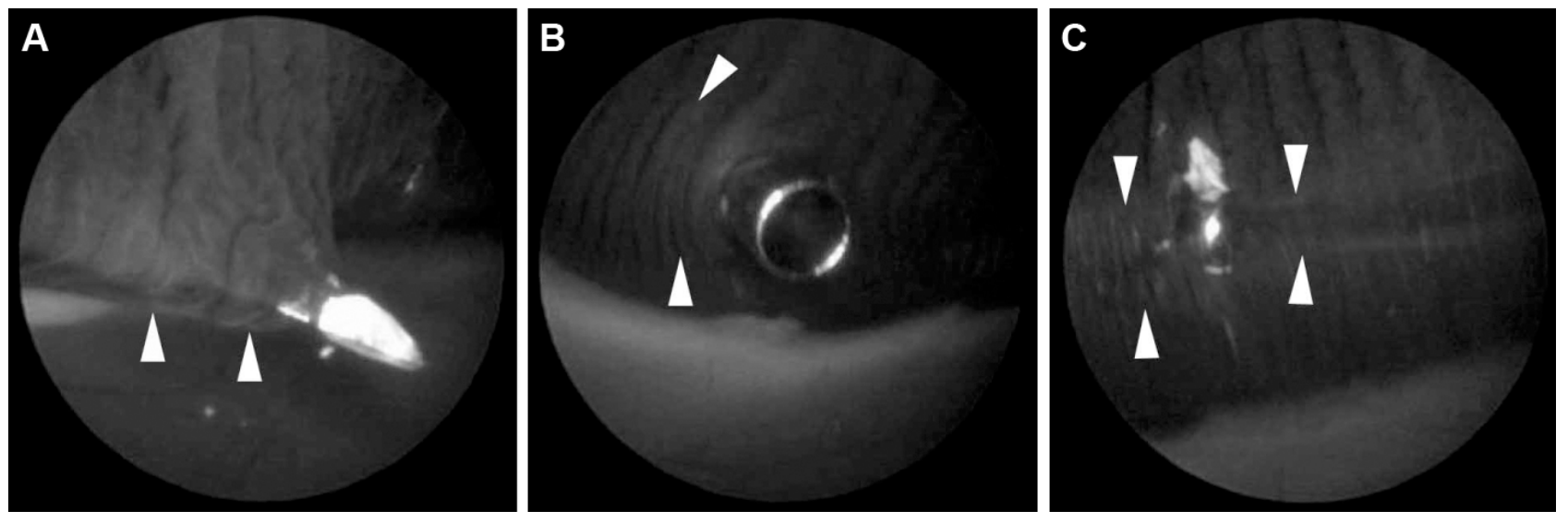

Figure 6 Endoscopic view of inner sclerotomy with 23-gauge ridged microvitreoretinal trocar cannula system.

Notes: (A) A stretched ciliary epithelium (white arrowheads) was observed during the insertion of the blade. (B) The stretched portion of the ciliary epithelium was folded (white arrowheads) after the blade was removed. (C) The incarceration of vitreous (white arrowheads) around a vitreous base was observed after removal of the cannula.

sites. The 23-gauge round-tipped beveled and pointed beveled blades created thicker incisions at the exit sites than at the entry sites, indicating more three-dimensional longitudinal wound architecture compared with both MVR-type blades. A quadrilateral facet was observed near the tip of the non-ridged MVR blade, suggesting that additional tip sharpening may have occurred during manufacturing. A sharper tip would facilitate incision creation, but the facet could cause the tissue to be cut unevenly, resulting in the nonlinear "M"-shaped incision observed in our incision architecture assessment. Surface striations were observed on non-ridged MVR, pointed beveled, and round-tipped beveled blades; these striations are likely artifacts introduced during manufacture and sharpening. Burrs were also evident on the cutting edge of the pointed beveled blade and could lead to creation of thicker incisions.

Oblique incisions made with non-ridged MVR blades in wound leakage testing were thicker at the entry site and had a lower leakage score (indicating more leakage at lower pressure) compared with oblique incisions made with ridged MVR blades. Wound leakage at low or moderate infusion pressures was observed with the non-ridged MVR blades, the pointed beveled blades, and the round-tipped beveled blades, suggesting unstable wound closure. Both the ridged and non-ridged MVR blades created thinner incisions at entry and exit sites. To achieve better wound closure, linear wound structure at the inner (exit) site is believed to be important for avoiding leakage of intraocular fluid and postoperative hypotony. ${ }^{25}$ The lack of wound leakage observed with ridged MVR blade incisions suggests that the linear slit-like incision made with these blades had more stable closure than the other incision shapes. This finding is consistent with previous reports that "V"-shaped or chevron-shaped incisions are associated with greater wound leakage and postoperative wound gaping. ${ }^{22,26}$

In a study of human eyes undergoing vitrectomy surgery, incisions created with MVR blades were linear-shaped and led to less postoperative wound gaping than chevron-shaped incisions created with a conventional blade. ${ }^{26}$ Several clinical studies have shown that MVR-style blades improve wound closure compared with beveled trocar cannulas. ${ }^{25-28}$ For example, the two-step sclerotomy method of initial penetration with an MVR blade followed by a blunt-tipped cannula inserter has been shown to reduce early postoperative hypotony ${ }^{27}$ and wound leakage ${ }^{25}$ compared with the beveled trocar cannula. In addition, the one-step methods with 23- and 25-gauge MVR blades have been reported to reduce postoperative hypotony in the early period compared with the beveled trocar. ${ }^{26,28}$ OCT images of the 23-gauge MVR blade incisions also showed earlier wound closure compared with beveled trocars. ${ }^{26}$ In a recent study of wound morphology in human eyes undergoing vitrectomy with incisions created using a 23- or 25-gauge ridged MVR blade, both gauge MVR blades led to complete wound closure and no leakage; ${ }^{29}$ our results are in general agreement with these findings. Together, these studies suggest that the morphologic differences among incisions created with trocar blades of different designs may influence wound closure.

Previous endoscopic evaluations showed that vitreous blocked the inner lips of the sclerotomy ports after conventional 23- and 25-gauge vitrectomy in uncomplicated cases of vitreous hemorrhage due to diabetic retinopathy. ${ }^{30}$ Our endoscopic observations had similar findings of vitreous incarceration at the inner sclerotomy sites. The incarcerated vitreous may act as a plug and help to seal the wound, preventing fluid leakage. In a recent study of wound closure after 25-gauge 
vitrectomy, swept-source OCT did not find significantly better rates of self-sealing and faster recovery of sclerotomies made with 25-gauge MVR blades compared with beveled blades. ${ }^{31}$ Thus, the MVR-type blades may have created a better wound structure, but it is possible that variations in incarcerated vitreous at the inner sclerotomy sites caused an unevenness in the self-sealing of sclerotomies that is independent of the wound structure. In the current study, we observed vitreous incarceration and similar wound closing with all blades tested and a lack of wound leakage with ridged MVR blades only; observed wound leakage with the non-ridged MVR, pointed beveled, and round-tipped beveled blades may therefore be related to effects of blade architecture on incarcerated vitreous and evenness of wound closure.

Together, the SEM image analysis, straight and oblique incision measurements, and wound leakage results suggest that the unilateral front-to-back and bilateral symmetry of the ridged MVR blades resulted in the linear, slit-like incisions observed for both straight and oblique angles. The combination of thinner thickness and smaller width of oblique incisions contributed to the incision architecture, which was associated with minimized wound leakage in the presence of increasing infusion pressure in porcine eyes. These findings are consistent with the results of a previous study showing that smaller-diameter oblique sclerotomies offered significantly more mechanical resistance to fluid flow at higher intraocular pressure in vitrectomized porcine eyes. ${ }^{32}$ Reduced wound leakage, similar to that observed with MVR blades in the current study and in the reports described above, may reduce the incidence of postoperative hypotony and endophthalmitis in a clinical setting.

A limitation of the study is that a silicone disc and porcine eyes were used to model human tissue; the results described here may not translate directly to clinical outcomes because of differences between human eyes and our model systems. The wound leakage and endoscopy experiments were performed in a relatively small number of porcine eyes using oblique incisions only; therefore, further evaluations may be needed. Furthermore, the lowest and highest infusion pressures used to evaluate wound leakage ( $5 \mathrm{mmHg}$ and $\geq 120 \mathrm{mmHg}$, respectively) may not be observed in routine MIVS; however, the pressures used in this study enabled assessment of incision sealing across a clinically relevant range of potential intraoperative pressures. Additionally, factors other than incision geometry (eg, lack of conjunctival displacement, patient-induced wound distortion) that may influence wound leakage ${ }^{3}$ were not addressed in the current study. These issues and the unmasked nature of this study may limit the strength of conclusions that can be inferred from our results.

\section{Conclusion}

In summary, the shape of the incisions and SEM analysis showed distinct differences in the architecture of the incisions, depending on the blade design and incision geometry. No leakage was observed with the incisions made using ridged MVR blades. The linear slit-like incisions generated with the ridged and non-ridged MVR-style blades were generally smaller at entry and exit sites and had more linear wound apposition compared with those generated by the beveled blades. Endoscopic assessment showed vitreous incarceration with all trocar cannula systems. These laboratory results highlight the differences among existing entry systems and suggest that ridged MVR blades may maximize the benefits of vitrectomy by minimizing wound leakage.

\section{Acknowledgments}

The authors thank David C Buboltz, MBA, for his assistance in organizing this study. Scientific writing assistance was provided by Heather D Starkey, PhD, of Complete Healthcare Communications, Inc. (Chadds Ford, PA, USA).

\section{Disclosure}

DJKA is an Alcon employee and owns stock in Alcon Research, Ltd. MI and AH have no proprietary interests. Funding for this study and scientific writing support was funded by Alcon Research, Ltd., Irvine, CA, USA. The authors report no other conflicts of interest in this work.

\section{References}

1. Fujii GY, De Juan E Jr, Humayun MS, et al. A new 25-gauge instrument system for transconjunctival sutureless vitrectomy surgery. Ophthalmology. 2002;109(10):1807-1812; discussion 1813.

2. Aylward GW. Sutureless vitrectomy. Ophthalmologica. 2011;225(2): 67-75.

3. Kaiser RS, Prenner J, Scott IU, et al. The Microsurgical Safety Task Force: evolving guidelines for minimizing the risk of endophthalmitis associated with microincisional vitrectomy surgery. Retina. 2010;30(4):692-699.

4. Shinoda H, Shinoda K, Satofuka S, et al. Visual recovery after vitrectomy for macular hole using 25-gauge instruments. Acta Ophthalmol. 2008;86(2):151-155.

5. Eckardt C. Transconjunctival sutureless 23-gauge vitrectomy. Retina. 2005;25(2):208-211.

6. Akcay BI, Uyar OM, Akkan F, Eltutar K. Outcomes of 23-gauge pars plana vitrectomy in vitreoretinal diseases. Clin Ophthalmol. 2011;5: 1771-1776.

7. Ibarra MS, Hermel M, Prenner JL, Hassan TS. Longer-term outcomes of transconjunctival sutureless 25-gauge vitrectomy. Am J Ophthalmol. 2005;139(5):831-836.

8. López-Guajardo L, Pareja-Esteban J, Teus-Guezala MA. Oblique sclerotomy technique for prevention of incompetent wound closure in transconjunctival 25-gauge vitrectomy. Am J Ophthalmol. 2006;141(6): 1154-1156. 
9. Shimada H, Nakashizuka H, Mori R, Mizutani Y, Hattori T. 25-gauge scleral tunnel transconjunctival vitrectomy. Am J Ophthalmol. 2006; 142(5):871-873.

10. Taban M, Sharma S, Ventura AA, Kaiser PK. Evaluation of wound closure in oblique 23-gauge sutureless sclerotomies with visante optical coherence tomography. Am J Ophthalmol. 2009;147(1):101-107.e101.

11. Taban M, Ventura AA, Sharma S, Kaiser PK. Dynamic evaluation of sutureless vitrectomy wounds: an optical coherence tomography and histopathology study. Ophthalmology. 2008;115(12):2221-2228.

12. Kunimoto DY, Kaiser RS, Wills Eye Retina Service. Incidence of endophthalmitis after 20- and 25-gauge vitrectomy. Ophthalmology. 2007; 114(12):2133-2137.

13. Scott IU, Flynn HW Jr, Dev S, et al. Endophthalmitis after 25-gauge and 20-gauge pars plana vitrectomy: incidence and outcomes. Retina. 2008;28(1):138-142.

14. Oshima Y, Kadonosono K, Yamaji H, et al. Multicenter survey with a systematic overview of acute-onset endophthalmitis after transconjunctival microincision vitrectomy surgery. Am J Ophthalmol. 2010;150(5): 716-725.e711.

15. Scott IU, Flynn HW Jr, Acar N, et al. Incidence of endophthalmitis after 20-gauge vs 23-gauge vs 25-gauge pars plana vitrectomy. Graefes Arch Clin Exp Ophthalmol. 2011;249(3):377-380.

16. Shimada H, Nakashizuka H, Hattori T, Mori R, Mizutani Y, Yuzawa M. Incidence of endophthalmitis after 20- and 25-gauge vitrectomy causes and prevention. Ophthalmology. 2008;115(12):2215-2220.

17. Gupta OP, Maguire JI, Eagle RC Jr, Garg SJ, Gonye GE. The competency of pars plana vitrectomy incisions: a comparative histologic and spectrophotometric analysis. Am J Ophthalmol. 2009;147(2):243-250.e1.

18. Singh RP, Bando H, Brasil OF, Williams DR, Kaiser PK. Evaluation of wound closure using different incision techniques with 23-gauge and 25-gauge microincision vitrectomy systems. Retina. 2008;28(2): 242-248.

19. Acar N, Kapran Z, Unver YB, Altan T, Ozdogan S. Early postoperative hypotony after 25-gauge sutureless vitrectomy with straight incisions. Retina. 2008;28(4):545-552.

20. Singh A, Chen JA, Stewart JM. Ocular surface fluid contamination of sutureless 25-gauge vitrectomy incisions. Retina. 2008;28(4):553-557.

21. Woo SJ, Park KH, Hwang JM, Kim JH, Yu YS, Chung H. Risk factors associated with sclerotomy leakage and postoperative hypotony after 23-gauge transconjunctival sutureless vitrectomy. Retina. 2009;29(4): $456-463$.
22. Arevalo JF, Berrocal MH, Arias JD, Banaee T. Minimally invasive vitreoretinal surgery: is sutureless vitrectomy the future of vitreoretinal surgery? J Ophthalmic Vis Res. 2011;6(2):136-144.

23. Kopani KR, Page MA, Holiman J, Parodi A, Iliakis B, Chamberlain W. Femtosecond laser-assisted keratoplasty: full and partial-thickness cut wound strength and endothelial cell loss across a variety of wound patterns. Br J Ophthalmol. 2014;98(7):894-899.

24. Teuma EV, Bott S, Edelhauser HF. Sealability of ultrashort-pulse laser and manually generated full-thickness clear corneal incisions. J Cataract Refract Surg. 2014;40(3):460-468.

25. Choi KS, Kim HD, Lee SJ. Sclerotomy site leakage according to wound shape in 23-gauge microincisional vitrectomy surgery. Curr Eye Res. 2010;35(6):499-504.

26. Inoue M, Shinoda K, Hirakata A. Twenty-three gauge cannula system with microvitreoretinal blade trocar. Br J Ophthalmol. 2010;94(4):498-502.

27. Inoue M, Shinoda K, Shinoda H, Kawamura R, Suzuki K, Ishida S. Twostep oblique incision during 25-gauge vitrectomy reduces incidence of postoperative hypotony. Clin Experiment Ophthalmol. 2007;35(8):693-696.

28. Inoue M, Shinoda K, Shinoda H, Suzuki K, Kawamura R, Ishida S. 25 -gauge cannula system with microvitreoretinal blade trocar. Am J Ophthalmol. 2007;144(2):302-304.

29. Nagpal M, Paranjpe G, Mehrotra N, Bhardwaj S. Evaluation of wound morphology of sclerotomy sites of sutureless vitrectomy using spectralis anterior segment optical coherence tomography. Asia-Pacific Journal of Ophthalmology. 2014;3:88-93.

30. Nagpal M, Wartikar S, Nagpal K. Comparison of clinical outcomes and wound dynamics of sclerotomy ports of 20, 25, and 23 gauge vitrectomy. Retina. 2009;29(2):225-231.

31. Yamane S, Takemae K, Inoue M, et al. Evaluation of microincision vitrectomy wounds made with microvitreoretinal blade or beveled trocar by swept source optical coherence tomography. Retina. 2012;32(1):140-145.

32. Lopez-Guajardo L, Benitez-Herreros J, Silva-Mato A. Experimental model to evaluate mechanical closure resistance of sutureless vitrectomy sclerotomies using pig eyes. Invest Ophthalmol Vis Sci. 2011;52(7): 4080-4084.
Clinical Ophthalmology

\section{Publish your work in this journal}

Clinical Ophthalmology is an international, peer-reviewed journal covering all subspecialties within ophthalmology. Key topics include: Optometry; Visual science; Pharmacology and drug therapy in eye diseases; Basic Sciences; Primary and Secondary eye care; Patient Safety and Quality of Care Improvements. This journal is indexed on Submit your manuscript here: http://www.dovepress.com/clinical-ophthalmology-journal

\section{Dovepress}

PubMed Central and CAS, and is the official journal of The Society of Clinical Ophthalmology (SCO). The manuscript management system is completely online and includes a very quick and fair peer-review system, which is all easy to use. Visit http://www.dovepress.com/ testimonials.php to read real quotes from published authors. 